\title{
LINC00858 promotes retinoblastoma cell proliferation, migration and invasion by inhibiting miR-3182
}

\author{
QI WANG* ${ }^{*}$ YANNI ZHU*, GUOJIN ZUO, XIAOMING CHEN, JINKUI CHENG and SHU ZHANG \\ Department of Ophthalmology, The First Affiliated Hospital of Yangtze University, Jingzhou, Hubei 434000, P.R. China
}

Received April 13, 2019; Accepted October 2, 2019

DOI: $10.3892 /$ etm.2019.8294

\begin{abstract}
The aim of the present study was to determine the role of long intergenic non-protein coding RNA 858 (LINC00858) in retinoblastoma (RB) and investigate the underlying molecular mechanisms. RB tissues and paracancerous tissues of $27 \mathrm{RB}$ cases were obtained. RB cell lines (SO-RB50, Y79, HXO-RB44 and WERI-Rb1) and a normal retinal epithelial cell line (ARPE-19) were cultured for in vitro experiments. Batches of SO-RB50 and Y79 cells were assigned to groups transfected with small interfering RNA targeting LINC00858 (si-LINC00858 group), microRNA (miR)-3182 mimics or inhibitor, or the respective controls. A Cell Counting Kit-8 and Transwell assays were performed to assess the effect of the transfections on the proliferation, migration and invasion of SO-RB50 and Y79 cells. A luciferase reporter assay was performed using SO-RB50 cells to demonstrate the direct binding of LINC00858 and miR-3182. Reverse transcription-quantitative PCR was employed to detect LINC00858 and miR-3182 expression. Pearson correlation analysis was used to assess the correlation between the expression of LINC00858 and miR-3182. The results indicated that RB tissues and cells exhibited aberrantly elevated LINC00858 expression $(\mathrm{P}<0.05)$. Compared with those in the control-transfected group, SO-RB50 and Y79 cells of the si-LINC00858 group had a lower cell proliferation, as well as a lower number of migrated and invaded cells (all $\mathrm{P}<0.05$ ). miR-3182 was proven to be a target gene of LINC00858, to be abnormally downregulated in RB tissues and cells $(\mathrm{P}<0.05)$ and to be negatively correlated with LINC00858 expression. Compared with those in the si-LINC00858+ inhibitor-negative control group, SO-RB50 and Y79 cells of the si-LINC00858 + miR-3182 inhibitor group exhibited a
\end{abstract}

Correspondence to: Professor Yanni Zhu, Department of Ophthalmology, The First Affiliated Hospital of Yangtze University, 8 Airway Road, Shashi, Jingzhou, Hubei 434000, P.R. China

E-mail: yannizhuaaa@163.com

${ }^{*}$ Contributed equally

Key words: retinoblastoma, LINC00858, microRNA-3182, proliferation, invasion significantly higher relative proliferation, migration and invasion (all $\mathrm{P}<0.05)$. In conclusion, LINC00858 promoted RB cell proliferation, migration and invasion, at least partially by inhibiting miR-3182.

\section{Introduction}

Retinoblastoma (RB) is an intraocular malignancy that occurs in every 14,000-20,000 newborns (1). In spite of extensive research efforts made during the past few decades, the RB remains difficult to diagnose and cure, and the associated mortality is as high as $\sim 70 \%$ in developing countries (2). Early treatment is important for improving the prognosis but $\mathrm{RB}$ is difficult to detect at the initial stage, thereby leading to delays beyond the time-window for efficient treatment. Elucidation of the molecular mechanisms of the pathogenesis is important for early detection and targeted therapy for RB.

Long non-coding RNAs (lncRNAs) are a class of RNAs consisting of $>200$ nucleotides in length, which do not have the function of encoding proteins (3). However, they have been identified to regulate the development of a variety of diseases, particularly multiple cancers, by interfering with the expression of other genes $(4,5)$. Cumulative studies have indicated that certain IncRNAs are implicated in the genesis and progression of RB (6). As one of the earliest lncRNAs discovered, H19 was reported to be downregulated in RB tissues and cells, and it has been indicated that enhancing the expression of H19 may be a potential targeted therapeutic strategy for RB (6).

LINC00858, a member of the 1ncRNA family with 2,685 nucleotides in length, was only recently begun to be investigated. Previous studies have indicated that LINC00858 has roles in several tumor types, including lung cancer (7), colorectal cancer (8) and osteosarcoma (9). To the best of our knowledge, LINC00858 has not been previously investigated in RB. Therefore, the present study aimed to assess the expression of LINC00858 in RB and determine the underlying molecular mechanisms. The present study provides a potential novel therapeutic target for RB.

\section{Materials and methods}

Tissue sample collection. A total of 27 retinoblastoma (RB) patients (age, $49 \pm 6.7$ years; sex, 19 males and 8 females) were enrolled in the present study between July 2014 and May 2017. All patients were diagnosed with RB for the first time at The 
First People's Hospital of Jingzhou City (Jingzhou, China). During surgery, RB tissues and paracancerous normal tissues of all patients were collected. All tissue samples were kept in liquid nitrogen for subsequent analysis.

Written informed consent from all patients was provided and the study was approved by the Ethics Committee of The First People's Hospital of Jingzhou City (Jingzhou, China).

Cell lines and culture. RB cell lines (SO-RB50, Y79, HXO-RB44 and WERI-Rb1) and a normal retinal epithelial cell line (ARPE-19) were maintained in Dulbecco's modified Eagle's medium (DMEM; HyClone; GE Healthcare Life Sciences) containing $10 \%$ fetal bovine serum (FBS; HyClone; GE Healthcare Life Sciences) with penicillin (100 U/ml) and streptomycin $(100 \mathrm{~g} / \mathrm{ml})$ in sterile cell culture flasks and utilized for experiments as previously reported $(10,11)$. All cell lines were obtained from the Cell Bank of the Chinese Academy of Sciences (Shanghai, China). Cells were cultured as previously described (12). SO-RB50 cells were cultured for $<10$ passages. All cells were cultured in an incubator at $37^{\circ} \mathrm{C}$ in an atmosphere containing $5 \% \mathrm{CO}_{2}$. For use in the experiments, third-generation cells were collected, transfected as described below and inoculated into 6 -well plates ( $1 \times 10^{5}$ cells per well) for $48 \mathrm{~h}$.

Cell transfection. Transfections were performed using a Lipofectamine 2000 transfection kit (Thermo Fisher Scientific, Inc.). SO-RB50 and Y79 cells suspended in serum-free DMEM were inoculated in 6-well plates to undergo transfection with LINC00858 small interfering (si)RNA (100 nM; si-LINC00858 group; 5'-UUCAACUUAUGAAUUUGA GGG-3'), LINC00858 siRNA negative control (100 nM; si-NC group; 5'-AAUUCUCCGAACGUGUCACGU-3'), microRNA (miR)-3182 mimics (50 nM; miR-3182 group; 5'-CUGAUG UGAUGUCUUCG-3'), miR-3182 mimics negative control (50 nM; miR-NC group; 5'-UCACAACCUCCUAGAAAG AGUAGA-3'), miR-3182 inhibitor (50 nM; miR-3182 inhibitor group; 5'-GACUACACUACAGAAGC-3') or miR-3182 inhibitor negative control $(50 \mathrm{nM}$; inhibitor-NC group; 5 '-GCG UAACUAAUACAUCGGAUUCGU-3'), all of which were purchased from Shanghai GenePharma Co. Ltd. In addition, cells were subjected to co-transfection by using LINC00858 siRNA negative control and miR-3182 inhibitor negative control (si-NC + inhibitor-NC group), LINC00858 siRNA and miR-3182 inhibitor negative control (si-LINC00858 + inhibitor-NC group), and LINC00858 siRNA and miR-3182 inhibitor (si-LINC00858 + miR-3182 inhibitor group). Transfection efficiency was verified using reverse transcription-quantitative PCR (RT-qPCR) $48 \mathrm{~h}$ following transfection.

Cell Counting Kit-8 (CCK-8) assay. The viability of SO-RB50 and Y79 cells was detected using a CCK- 8 assay. In short, cells in the logarithmic growth phase were prepared as cell suspensions ( $1 \times 10^{5}$ cells $\left./ \mathrm{ml}\right)$ using DMEM containing $10 \%$ FBS. A total of $100 \mu 1$ of the cell suspension was inoculated into 96-well plates. All plates were incubated at $37^{\circ} \mathrm{C}$ with $5 \% \mathrm{CO}_{2}$ for 24,48 or $72 \mathrm{~h}$ following transfection. Then $10 \mu \mathrm{l} \mathrm{CCK-8} \mathrm{solution} \mathrm{was} \mathrm{added} \mathrm{to} \mathrm{each}$ well, incubated for $2 \mathrm{~h}$ at $37^{\circ} \mathrm{C}$ and the absorbance of each well was measured by using a microplate reader (Tecan Group, Ltd.) at a wavelength of $450 \mathrm{~nm}$.
Transwell experiment. Suspensions of SO-RB50 and Y79 cells in serum-free DMEM $\left(100 \mu 1,1 \times 10^{5}\right.$ cells $\left./ \mathrm{ml}\right)$ were added into Transwell inserts $\left(8-\mu \mathrm{m}\right.$ pores) pre-coated with Matrigel ${ }^{\circledR}$. All Transwell inserts were placed in 6-well plates with $1 \mathrm{ml}$ DMEM (10\% FBS) at the bottom of each well. These plates were incubated at $37^{\circ} \mathrm{C}$ with $5 \% \mathrm{CO}_{2}$ for $48 \mathrm{~h}$. Cells that failed to penetrate the membrane were carefully scraped off with a cotton swab. The invaded cells at the lower side of the membrane were then fixed with $4 \%$ formaldehyde for $5 \mathrm{~min}$ at room temperature, stained with crystal violet $(0.1 \%)$ for $10 \mathrm{~min}$ and counted in 5 randomly selected non-overlapping fields under a microscope. The cell migration ability was also detected using the same procedure as above, but no Matrigel was placed on the Transwell inserts.

Luciferase reporter gene assay. Using a bioinformatics tool (miRDB; http://mirdb.org/miRDB/index.html), miR-3182 was predicted as a target of LINC00858. The LINC00858 wild-type (Wt) sequence with the microRNA (miR)-3182 binding site and the respective mutant-type (Mut) sequence were synthesized by Shanghai Jima Gene Co., Ltd. These sequences were each inserted into pmirGLO Dual-luciferase vector by the XhoI and XbaI restriction sites. SO-RB50 cells of the miR-NC $(50 \mathrm{nM})$ and miR-3182 groups $(50 \mathrm{nM})$ were subjected to co-transfection with luciferase reporter vector $(1 \mu \mathrm{g})$ encoding the LINC00858 Wt sequence or Mut sequence. After transfection, all cells were maintained in DMEM with $10 \% \mathrm{FBS}$ at $37^{\circ} \mathrm{C}$ with $5 \% \mathrm{CO}_{2}$ for $48 \mathrm{~h}$. A Dual-luciferase Reporter Assay Kit (Promega Corp.) was used to detect the luciferase activity. Renilla luciferase activity was detected for normalization.

$R T$ - $q P C R$. Total RNA was extracted from cells/tissues by using TRIzol ${ }^{\circledast}$. RT was performed by using $30 \mu \mathrm{g}$ samples according to the protocol of TaqMan ${ }^{\mathrm{TM}}$ MicroRNA Reverse Transcription Kit (Thermo Fisher Scientific, Inc.). LINC00858 and miR-3182 expression was determined by using an ABI7500 qPCR machine (Applied Biosystems; Thermo Fisher Scientific, Inc.) with the following thermocycling program: Pre-denaturation at $95^{\circ} \mathrm{C}$ for $5 \mathrm{~min}$, followed by 38 cycles of denaturation at $95^{\circ} \mathrm{C}$ for $15 \mathrm{sec}$, annealing at $60^{\circ} \mathrm{C}$ for $30 \mathrm{sec}$ and elongation at $72^{\circ} \mathrm{C}$ for $30 \mathrm{sec}$. The following primers were used: LINC00858 forward, 5'-CCCAGCTCC TTACACACGTT-3' and reverse, 5'-TTCAGAGGCCTGCAT CACTG-3'; miR-3182 forward, 5'-CACTCAGCTGGCTTC TGTAGTG-3' and reverse, 5'-CTGGTGTCGTGGAGTCG-3'; U6, forward, 5'-CTCGCTTCGGCAGCACATATACT-3' and reverse, 5'-ACGCTTCACGAATTTGCGTGTC-3'. Relative LINC00858 and miR-3182 expression was normalized to U6 and the $2^{-\Delta \Delta \mathrm{Cq}}$ method was used for quantification (13).

Statistical analysis. SPSS 17.0 statistical software (SPSS, Inc.) was used to process the data. All measurement data were expressed as the mean \pm standard deviation. A Student's t-test was used to analyze differences between two groups. One-way analysis of variance followed by Tukey's post-hoc test were used for multiple comparisons. Pearson's correlation analysis was selected to assess the correlation between two genes. $\mathrm{P}<0.05$ was considered to indicate statistical significance. All experiments were performed at least 3 times independently. 

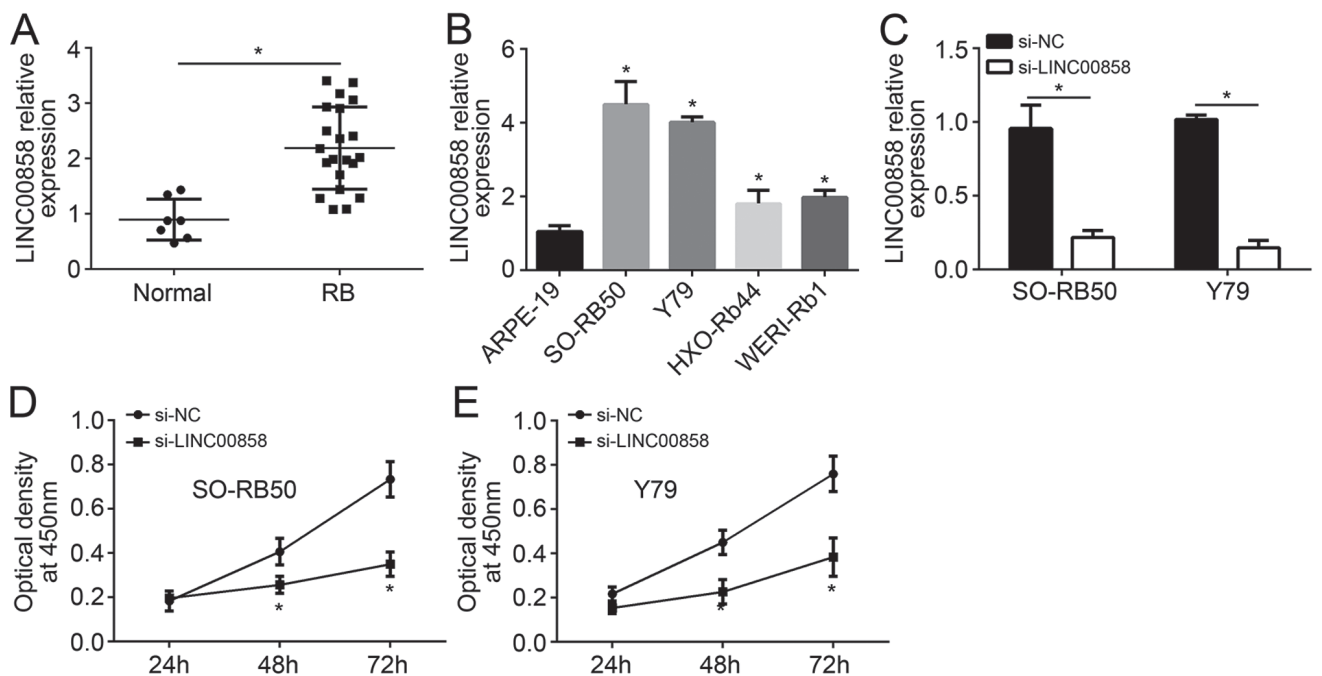

Figure 1. LINC00858 is upregulated in RB tissues and cell lines, while knockout of LINC00858 inhibits RB cell proliferation. (A) Compared with that in normal paracancerous tissues, the relative expression of LINC00858 was significantly upregulated in RB tissues. (B) Increased relative LINC00858 expression was detected in RB cell lines (SO-RB50, Y79, HXO-RB44 and WERI-Rb1) when compared with that in a normal retinal epithelial cell line (ARPE-19). (C) SO-RB50 and Y79 cells of the si-LINC00858 group had a significantly lower relative LINC00858 expression than cells of the si-NC group. (D) Cell Counting Kit- 8 assay showed that at 48 and $72 \mathrm{~h}$ of culture, SO-RB50 cells of the si-LINC00858 group had obviously lower optical density values at $450 \mathrm{~nm}$ than cells of the si-NC group. (E) Cell Counting Kit-8 assay showed that at 48 and $72 \mathrm{~h}$ of culture, Y79 cells in the si-LINC00858 group exhibited lower optical density values at $450 \mathrm{~nm}$ compared with cells in the si-NC group. " $\mathrm{P}<0.05$. RB, retinoblastoma; LINC00858, long intergenic non-protein coding RNA 858 ; si-LINC00858, small interfering RNA targeting LINC00858; si-NC, scrambled control.

\section{Results}

LINC00858 is upregulated in RB tissues and cells and knockout of LINC00858 inhibits RB cell proliferation. The expression levels of LINC00858 in RB tissues and paracancerous normal tissues of $27 \mathrm{RB}$ cases were detected. Compared with the relative expression of LINC00858 in paracancerous normal tissues, it was markedly elevated in RB tissues $(\mathrm{P}<0.05$; Fig. 1A). The expression of LINC00858 in RB cells was also assessed, and significantly increased relative LINC00858 expression was identified in the RB cell lines SO-RB50, Y79, HXO-RB44 and WERI-Rb1 when compared with that in the normal retinal epithelial cell line ARPE-19 (P<0.05; Fig. 1B). The SO-RB50 and Y79 cell lines were used for the subsequent studies, as these two cell lines had the highest expression levels of LINC00858 among the 4 RB cell lines. After transfection, SO-RB50 and Y79 cells of the si-LINC00858 group had a significantly lower relative LINC00858 expression than cells of the si-NC group $(\mathrm{P}<0.05$; Fig. 1C). Furthermore, at 48 and $72 \mathrm{~h}$ of culture, SO-RB50 and Y79 cells of the si-LINC00858 group had an obviously lower number of viable cells than those of the si-NC group $(\mathrm{P}<0.05$; Fig. 1D and $\mathrm{E})$.

Knockdown of LINC00858 inhibits RB cell migration and invasion. The Transwell assay, an effective method for detecting cell migration and invasion, was performed in the present study. As indicated in Fig. 2A, the number of migrated SO-RB50 and Y79 cells in the si-LINC00858 group was markedly decreased when compared with that in the si-NC group $(\mathrm{P}<0.05)$. A similar result was also obtained in the cell invasion assay, as SO-RB50 and Y79 cells of the si-LINC00858 group had an obviously lower number of invaded cells compared with those in the si-NC group ( $\mathrm{P}<0.05$; Fig. $2 \mathrm{~B})$.
miR-3182 is a downstream target gene of LINC00858. The LINC00858-WT sequence and -Mut sequence containing the miR-3182 binding sites were synthesized and the predicted binding sites are indicated in Fig. 3A. SO-RB50 and Y79 cells of the miR-3182 group exhibited much higher miR-3182 relative expression than those of the miR-NC group $(\mathrm{P}<0.05)$, confirming that SO-RB50 and Y79 cells were successfully transfected with the mimics (Fig. 3B). In the group of SO-RB50 cells co-transfected with the reporter plasmid carrying the LINC00858-Mut sequence and miR-3182 mimics, the relative luciferase activity was not obviously changed compared with that in the LINC00858-Mut plasmid + miR-NC group. However, if the reporter vector carrying the LINC00858-WT sequence was used, significantly reduced relative luciferase activity was observed in the group co-transfected with miR-3182 mimics when compared with that in the group co-transfected with miR-NC ( $\mathrm{P}<0.05$; Fig. 3C). In addition, SO-RB50 and Y79 cells of the si-LINC00858 group had a significantly higher relative miR-3182 expression when compared with that of the si-NC group ( $\mathrm{P}<0.05$; Fig. 3D), and significantly lower relative LINC00858 expression was observed in SO-RB50 and Y79 cells of the miR-3182 group when compared to that in the miR-NC group $(\mathrm{P}<0.05$; Fig. 3E).

miR-3182 is negatively correlated with LINC00858 expression in RB tissues. miR-3182 expression levels were measured in $\mathrm{RB}$ cells and tissues. When compared with that in ARPE-19 cells, the relative expression of miR-3182 was significantly declined in RB cell lines (SO-RB50, Y79, HXO-RB44 and WERI-Rb1; $\mathrm{P}<0.05$; Fig. 4A). Furthermore, compared with that in paracancerous normal tissues, the relative expression of $\mathrm{miR}-3182$ in $\mathrm{RB}$ tissues was also markedly reduced ( $\mathrm{P}<0.05$; Fig. 4B). Pearson correlation analysis indicated that miR-3182 expression was negatively correlated with LINC00858 expression in RB tissues (Fig. 4C). 

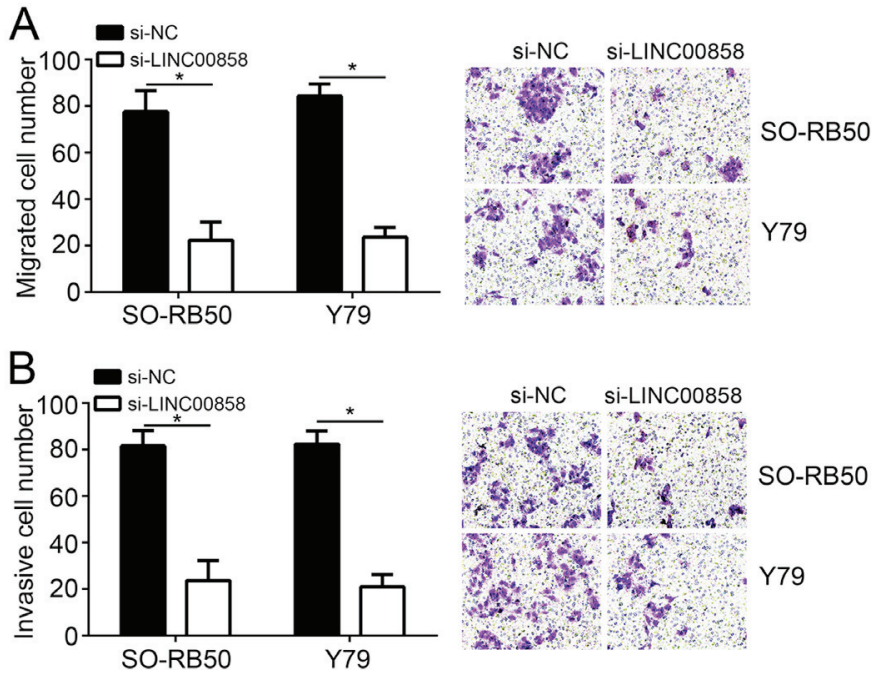

Figure 2. Knockout of LINC00858 inhibits retinoblastoma cell migration and invasion. (A) The number of migrated SO-RB50 and Y79 cells of the si-LINC00858 group was significantly reduced when compared with that of the si-NC group. Magnification, x100. (B) SO-RB50 and Y79 cells of the si-LINC00858 group had an obviously lower number of invasive cells compared with those in the si-NC group. Magnification, $\mathrm{x} 100$. " $\mathrm{P}<0.05$. LINC00858, long intergenic non-protein coding RNA 858; si-LINC00858, small interfering RNA targeting LINC00858; si-NC, scrambled control.
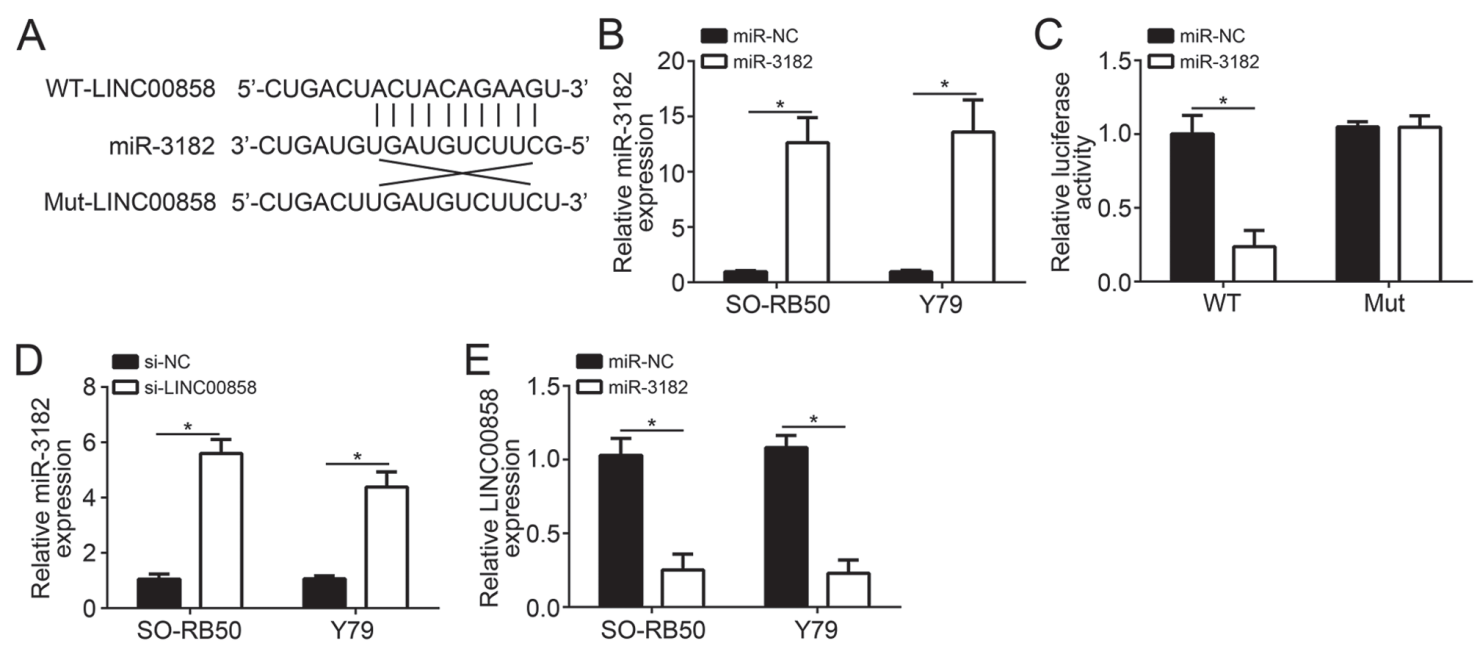

Figure 3. miR-3182 is a direct target of LINC00858. (A) LINC00858-WT sequence and -Mut sequence containing the miR-3182 binding sites. (B) SO-RB50 and Y79 cells of the miR-3182-transfected group exhibited higher relative miR-3182 expression than those of the miR-NC group. (C) Luciferase reporter assay showed that miR-3182 co-transfection inhibited the luciferase activity of the wt-LINC00858 reporter. (D) SO-RB50 and Y79 cells of the si-LINC00858 group had a significantly higher relative miR-3182 expression when compared with that of the si-NC group. (E) Lower relative LINC00858 expression was observed in SO-RB50 and Y79 cells of the miR-3182-transfected group when compared with that in the miR-NC group. ${ }^{*} \mathrm{P}<0.05$. LINC00858, long intergenic non-protein coding RNA 858; si-LINC00858, small interfering RNA targeting LINC00858; si-NC, scrambled control; miR, microRNA; miR-NC, miR mimics control; WT, wild-type; Mut, mutated.

Knockdown of LINC00858 inhibits the proliferation, migration and invasion of RB cells by promoting miR-3182. SO-RB50 and Y79 cells of the miR-3182 inhibitor group exhibited a much lower miR-3182 expression than cells of the inhibitor-NC group $(\mathrm{P}<0.05$; Fig. 5A), demonstrating that SO-RB50 and Y79 cells were effectively transfected. At $72 \mathrm{~h}$ after co-transfection, SO-RB50 and Y79 cells of the si-LINC00858 + inhibitor-NC group had a significantly lower relative proliferation than those of the si-NC + inhibitor-NC group $(\mathrm{P}<0.05)$. However, when compared with the relative proliferation of SO-RB50 and Y79 cells in the si-LINC00858 + inhibitor-NC group at the same time-point, it was obviously increased in cells of the si-LINC00858 + miR-3182 inhibitor group $(\mathrm{P}<0.05$; Fig. 5B). The results of the Transwell assays indicated that, compared with those in the si-NC + inhibitor-NC group, the number of migrated and invaded SO-RB50 and Y79 cells in the si-LINC00858 + inhibitor-NC group was significantly decreased $(\mathrm{P}<0.05)$. However, a significantly increased number of migrated and invaded SO-RB50 and Y79 cells was observed in the si-LINC00858 + miR-3182 inhibitor group when compared with that in the si-LINC00858 + inhibitor-NC group $(\mathrm{P}<0.05$; Fig. 5C and D).

\section{Discussion}

In recent years, IncRNAs have been widely investigated as part of the molecular basis of various diseases and are a 

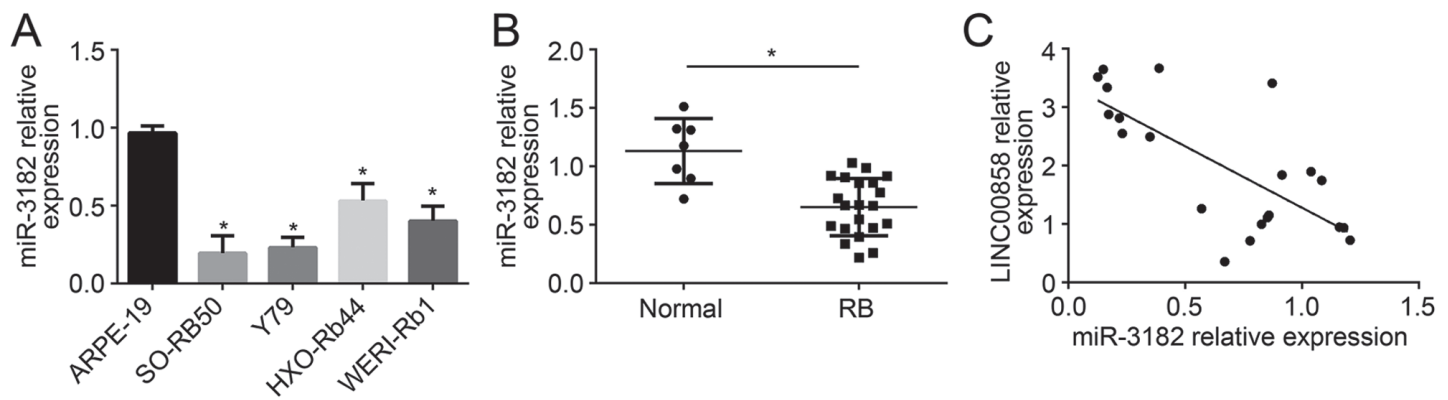

Figure 4. miR-3182 expression is negatively correlated with LINC00858 expression in RB tissues. (A) The relative miR-3182 expression in RB cell lines (SO-RB50, Y79, HXO-RB44 and WERI-Rb1) was much lower than that in a normal retinal epithelial cell line (ARPE-19). "P<0.05 vs. ARPE-19. (B) Relative miR-3182 expression in RB tissues was also markedly lower than that in normal tissues. " $\mathrm{P}<0.05$. (C) miR-3182 was negatively correlated with LINC00858 expression in RB tissues. $\mathrm{r}=-7239, \mathrm{P}<0.001$. RB, retinoblastoma; miR, microRNA; LINC00858, long intergenic non-protein coding RNA 858.
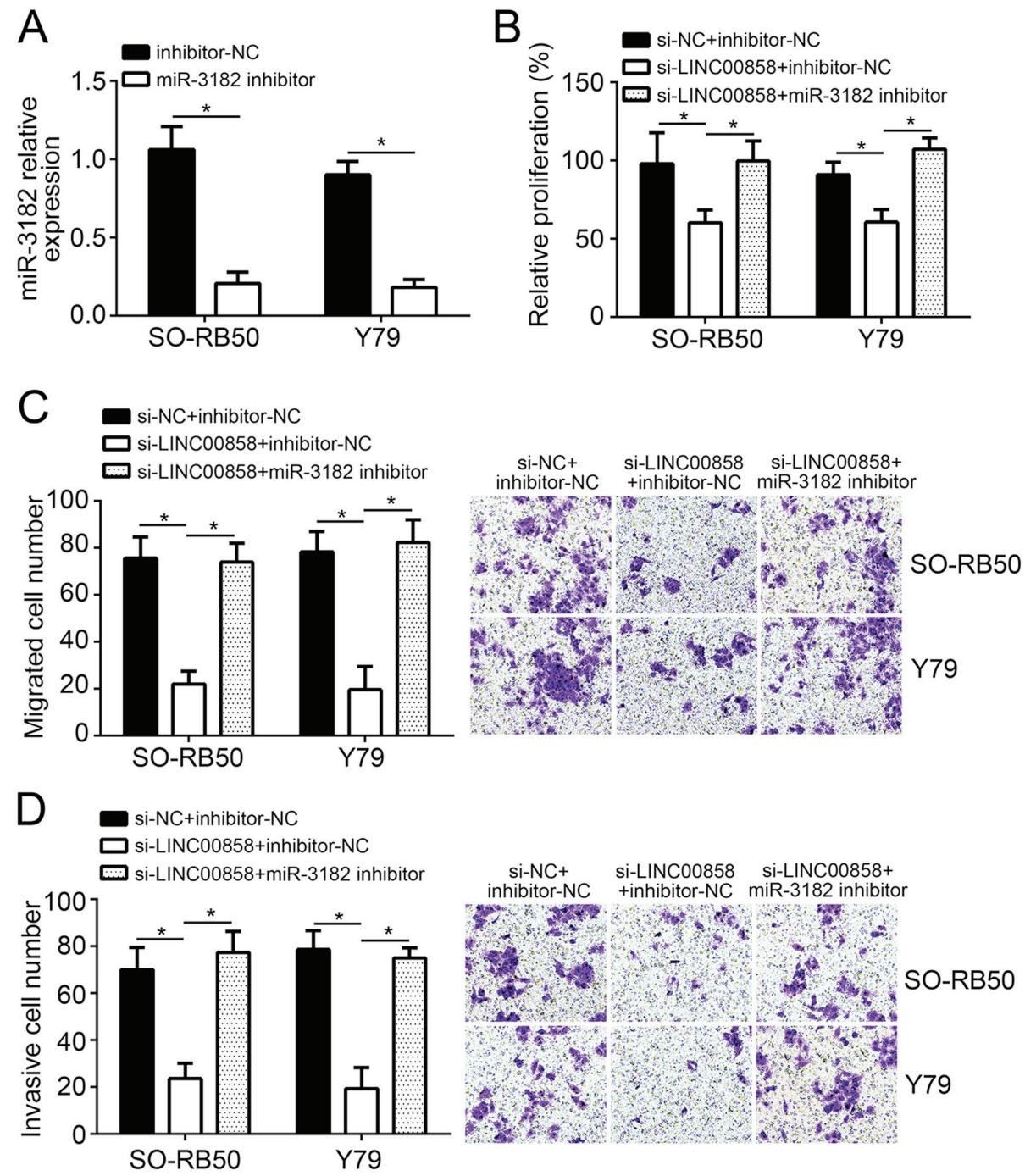

Figure 5. LINC00858 knockdown inhibits proliferation, migration and invasion of RB cells by promoting miR-3182. (A) SO-RB50 and Y79 cells of the miR-3182 inhibitor group exhibited much lower miR-3182 expression than cells of the inhibitor-NC group. (B) LINC00858 knockdown inhibited the proliferation of RB cells, which was reversed by co-transfection with miR-3182 inhibitor. (C and D) LINC00858 knockdown inhibited the migration and invasion of RB cells, which was reversed by miR-3182 inhibitor co-transfection. Magnification, $\mathrm{x} 100$. "P<0.05. LINC00858, long intergenic non-protein coding RNA 858; si-LINC00858, small interfering RNA targeting LINC00858; si-NC, scrambled control; RB, retinoblastoma; miR, microRNA; NC, negative control.

hot spot particularly in the field of tumors. They may exert cancer-promoting or anti-cancer effects by regulating the expression of various miRNAs and proteins at epigenetic, transcriptional and post-transcriptional levels $(14,15)$. LncRNAs are mainly transcribed by RNA polymerase II and were originally thought to have no biological functions (16). 
However, recent studies have proved that lncRNAs participate in several important regulatory processes, including chromatin modification, $\mathrm{X}$ chromosome silencing, transcriptional activation, transcriptional interference and intranuclear transport $(17,18)$. Although the biological functions and molecular mechanisms of most lncRNAs have remained to be fully defined, lncRNAs have been indicated to be involved in the regulation of cell differentiation, apoptosis, growth and development, as well as numerous human diseases $(19,20)$.

The lncRNA LINC00858 has been studied in human diseases in recent years. Increased LINC00858 expression was reported in non-small cell lung cancer, and its upregulation significantly enhanced non-small cell lung cancer cell proliferation, migration and invasion. Upregulated LINC00858 expression was identified to be closely linked to the progression of non-small cell lung cancer (21). Another study indicated that the expression level of LINC00858 in lung adenocarcinoma was 5.23 times that in normal lung tissues (22). Upregulated LINC00858 expression was also reported in osteosarcoma and knockdown of LINC00858 effectively inhibited osteosarcoma cell proliferation in vitro as well as tumor growth in vivo (9). The results of the present study also demonstrated abnormally upregulated LINC00858 expression in RB tissues and cells, which was similar to the results of these above previous studies. The present results also indicated that the in vitro proliferation, migration and invasion abilities of RB cells were significantly reduced after LINC00858 was knocked down.

One of the important ways for IncRNAs to participate in tumor regulation is to interfere with the expression of miRNAs. Studies have demonstrated that lncRNAs may act as an endogenous 'miRNA sponge' to inhibit miRNA expression. The binding of miRNAs to lncRNAs may lead to degradation of IncRNAs, thereby affecting the progression of tumors (23). In the present study, LINC00858 was demonstrated to exert its effects, at least partially, through inhibiting the expression of miR-3182 to promote RB cell proliferation, migration and invasion. miR-3182 was recently discovered to be involved in the regulation of certain human diseases. Razaviyan et al (24) illustrated that the expression level of miR-3182 was declined in triple-negative breast cancer. They discovered that miR-3182 may be an effective inhibitory agent for ribosomal protein S6 kinase B1 and mTOR. Thus, miR-3182 may serve as a potential target for the treatment of triple-negative breast cancer. Zhu et al (25) reported that the expression level of miR-3182 was markedly reduced in osteosarcoma tissues and five osteosarcoma cell lines, and compared with that in patients with higher miR-3182 expression, those with lower miR-3182 expression exhibited shorter overall survival. Through in-depth research, they indicated that miR-3182 may attenuate biological behaviors of osteosarcoma cells (including proliferation, migration and invasion) in vitro and inhibit osteosarcoma cell growth in vivo. Similar to the above studies, the present results also demonstrated decreased miR-3182 expression in RB tissues and cells, and indicated that miR-3182 may serve as a tumor suppressor gene in RB. Of note, the present study revealed that miR-3182 is a direct downstream target of LINC00858 and its expression was negatively regulated by LINC00858. These results provide an important theoretical basis for potential targeted therapies for RB.

In conclusion, the present study assessed the molecular mechanisms of LINC00858 in regulating the progression of
RB. LINC00858 was abnormally upregulated in RB tissues and cell lines, and LINC00858 knockdown suppressed RB cell proliferation, migration and invasion in vitro by enhancing the expression of miR-3182. LINC00858 may be a novel therapeutic target for RB and the present study provided an important theoretical basis for the targeted therapy of RB. Future studies should be focused on the role of LINC00858 in RB in vivo.

\section{Acknowledgements}

Not applicable.

\section{Funding}

No funding was received.

\section{Availability of data and materials}

All data generated or analyzed during this study are included in this published article.

\section{Authors' contributions}

QW and YZ contributed to the conception and design of the present study. In addition, YZ analyzed and interpreted the results and wrote the manuscript. QW, GZ, XC, JC and SZ performed the experiments. All authors read and approved the final manuscript.

\section{Ethics approval and consent to participate}

The present study was approved by The Ethics Committee of The First People's Hospital of Jingzhou City (Jingzhou, China). Written informed consent was obtained from all patients enrolled.

\section{Patient consent for publication}

Not applicable.

\section{Competing interests}

The authors declare that they have no competing interests.

\section{References}

1. Broaddus E, Topham A and Singh AD: Incidence of retinoblastoma in the USA: 1975-2004. Br J Ophthalmol 93: 21-23, 2009.

2. Dimaras H, Corson TW, Cobrinik D, White A, Zhao J, Munier FL, Abramson DH, Shields CL, Chantada GL, Njuguna F and Gallie BL: Retinoblastoma. Nat Rev Dis Primers 1: 15021, 2015.

3. Dolcino M, Pelosi A, Fiore PF, Patuzzo G, Tinazzi E, Lunardi C and Puccetti A: Long non-coding RNAs play a role in the pathogenesis of psoriatic arthritis by regulating MicroRNAs and genes involved in inflammation and metabolic syndrome. Front Immunol 9: 1533, 2018.

4. Mathy NW and Chen XM: Long non-coding RNAs (lncRNAs) and their transcriptional control of inflammatory responses. J Biol Chem 292: 12375-12382, 2017.

5. Kapinova A, Kubatka P, Zubor P, Golubnitschaja O, Dankova Z, Uramova S, Pilchova I, Caprnda M, Opatrilova R, Richnavsky J, et al: The hypoxia-responsive long non-coding RNAs may impact on the tumor biology and subsequent management of breast cancer. Biomed Pharmacother 99: 51-58, 2018. 
6. Zhang A, Shang W, Nie Q, Li T and Li S: Long non-coding RNA H19 suppresses retinoblastoma progression via counteracting miR-17-92 cluster. J Cell Biochem 119: 3497-3509, 2018.

7. Xue M, Shi D, Xu G and Wang W: The long noncoding RNA linc00858 promotes progress of lung cancer through miR-3182/MMP2 axis. Artif Cells Nanomed Biotechnol 47: 2091-2097, 2019

8. Sha QK, Chen L, Xi JZ and Song H: Long non-coding RNA LINC00858 promotes cells proliferation, migration and invasion by acting as a ceRNA of miR-22-3p in colorectal cancer. Artif Cells Nanomed Biotechnol 47: 1057-1066, 2019.

9. Gu Z, Hou Z, Zheng L, Wang X, Wu L and Zhang C: Long noncoding RNA LINC00858 promotes osteosarcoma through regulating miR-139-CDK14 axis. Biochem Biophys Res Commun 503: 1134-1140, 2018.

10. Zhong W, Yang J, Li M, Li L and Li A: Long noncoding RNA NEAT1 promotes the growth of human retinoblastoma cells via regulation of miR-204/CXCR4 axis. J Cell Physiol 234 11567-11576, 2019.

11. Wu XZ, Cui HP, Lv HJ and Feng L: Knockdown of IncRNA PVT1 inhibits retinoblastoma progression by sponging miR-488-3p. Biomed Pharmacother 112: 108627, 2019.

12. Meng B, Wang Y and Li B: Suppression of PAX6 promotes cell proliferation and inhibits apoptosis in human retinoblastoma cells. Int J Mol Med 34: 399-408, 2014.

13. Livak KJ and Schmittgen TD: Analysis of relative gene expression data using real-time quantitative PCR and the 2(-Delta Delta C(T)) method. Methods 25: 402-408, 2001

14. Lei R, Xue M, Zhang L and Lin Z: Long noncoding RNA MALAT1-regulated microRNA 506 modulates ovarian cancer growth by targeting iASPP. Onco Targets Ther 10: 35-46, 2016.

15. Liu W, Ma R and Yuan Y: Post-transcriptional regulation of genes related to biological behaviors of gastric cancer by long noncoding RNAs and MicroRNAs. J Cancer 8: 4141-4154, 2017.

16. Ponting CP, Oliver PL and Reik W: Evolution and functions of long noncoding RNAs. Cell 136: 629-641, 2009.
17. Mercer TR, Dinger ME and Mattick JS: Long non-coding RNAs: Insights into functions. Nat Rev Genet 10: 155-159, 2009

18. Wilusz JE, Sunwoo H and Spector DL: Long noncoding RNAs: Functional surprises from the RNA world. Genes Dev 23: 1494-1504, 2009.

19. Qiao L, Liu X, Tang Y, Zhao Z, Zhang J and Feng Y: Down regulation of the long non-coding RNA PCAT-1 induced growth arrest and apoptosis of colorectal cancer cells. Life Sci 188: 37-44, 2017.

20. Li A, Yan Q, Zhao X, Zhong J, Yang H, Feng Z, Du Y, Wang Y, Wang $\mathrm{Z}$, Wang $\mathrm{H}$, et al: Decreased expression of PBLD correlates with poor prognosis and functions as a tumor suppressor in human hepatocellular carcinoma. Oncotarget 7: 524-537, 2016.

21. Zhu SP, Wang JY, Wang XG and Zhao JP: Long intergenic non-protein coding RNA 00858 functions as a competing endogenous RNA for miR-422a to facilitate the cell growth in non-small cell lung cancer. Aging (Albany NY) 9: 475-486, 2017.

22. Xu G, Chen J, Pan Q, Huang K, Pan J, Zhang W, Chen J, Yu F, Zhou $\mathrm{T}$ and Wang Y: Long noncoding RNA expression profiles of lung adenocarcinoma ascertained by microarray analysis. PLoS One 9: e104044, 2014.

23. Li T, Meng XL and Yang WQ: Long noncoding RNA PVT1 acts as a 'Sponge' to inhibit microRNA-152 in gastric cancer cells. Dig Dis Sc 62: 3021-3028, 2017.

24. Razaviyan J, Hadavi R, Tavakoli R, Kamani F, Paknejad M and Mohammadi-Yeganeh S: Expression of miRNAs targeting mTOR and S6K1 genes of mTOR signaling pathway including miR-96, miR-557, and miR-3182 in triple-negative breast cancer. Appl Biochem Biotechnol 186: 1074-1089, 2018.

25. Zhu KP, Ma XL and Zhang CL: LncRNA ODRUL contributes to osteosarcoma progression through the miR-3182/MMP2 axis. Mol Ther 25: 2383-2393, 2017.

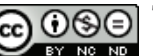

This work is licensed under a Creative Commons Attribution-NonCommercial-NoDerivatives 4.0 International (CC BY-NC-ND 4.0) License. 